\title{
Efficient synthesis of tension modulation in strings and membranes based on energy estimation
}

\author{
Federico Avanzini ${ }^{\mathrm{a})}$ and Riccardo Marogna \\ Department of Information Engineering, University of Padova, Via G. Gradenigo 6/A, IT-35131, Padova, Italy \\ Balázs Bank \\ Department of Measurement and Information Systems, Budapest University of Technology and Economics, \\ Budapest, Hungary
}

(Received 29 November 2010; revised 4 March 2011; accepted 6 March 2011)

\begin{abstract}
String and membrane vibrations cannot be considered as linear above a certain amplitude due to the variation in string or membrane tension. A relevant special case is when the tension is spatially constant and varies in time only in dependence of the overall string length or membrane surface. The most apparent perceptual effect of this tension modulation phenomenon is the exponential decay of pitch in time. Pitch glides due to tension modulation are an important timbral characteristic of several musical instruments, including the electric guitar and tom-tom drum, and many ethnic instruments. This paper presents a unified formulation to the tension modulation problem for onedimensional (1-D) (string) and two-dimensional (2-D) (membrane) cases. In addition, it shows that the short-time average of the tension variation, which is responsible for pitch glides, is approximately proportional to the system energy. This proportionality allows the efficient physics-based sound synthesis of pitch glides. The proposed models require only slightly more computational resources than linear models as opposed to earlier tension-modulated models of higher complexity. (C) 2012 Acoustical Society of America. [DOI: 10.1121/1.3651097]
\end{abstract}

PACS number(s): 43.75.Hi, 43.75.Mn [NHF]

Pages: 897-906

\section{INTRODUCTION}

The main features of string and membrane oscillation are well described by the one- and two-dimensional (1- and 2 -D, respectively) linear wave equation. However, some relevant secondary effects can only be accounted for by abandoning the hypothesis of linear vibration. In particular, the assumption of constant tension does not hold above a certain vibration amplitude because the tension depends on the displacement. This type of nonlinearity is termed "geometric nonlinearity" because it comes from the geometry of the problem (the elasticity of the material is assumed to be linear).

The effects of the geometric nonlinearity in strings are classified into different regimes, depending on the parameters of the string and excitation force. ${ }^{1}$ These are listed in Table I, showing the significance of transverse to longitudinal coupling $(\mathrm{T} \rightarrow \mathrm{L}$ ), longitudinal to transverse coupling $(\mathrm{L} \rightarrow \mathrm{T})$, and longitudinal inertial effects. Various models have been proposed ${ }^{1-3}$ to accurately simulate these regimes. However, they are computationally demanding, and their accuracy is not always required because, depending on the instrument considered, only a subset of nonlinear phenomena are perceptually relevant.

The tension modulation regime is found when the tension varies in time but it is spatially uniform along the string. This happens when the bandwidth of the nonlinear excitation force from the transverse vibration is below the longitudinal

\footnotetext{
a) Author to whom correspondence should be addressed. Electronic mail: avanzini@dei.unipd.it
}

modal frequencies, so that the inertial effects of the longitudinal motion are negligible and longitudinal motion immediately follows transverse motion to find an equilibrium in the force, resulting in uniform tension. Tension modulation is particularly relevant for certain instruments (e.g., electric and steel-stringed acoustic guitars and various ethnic instruments ${ }^{4}$ ), its most perceptually prominent effect being the decrease in pitch as the sound decays. In other cases, other phenomena may be relevant. As an example, for the piano, string longitudinal inertial effects come into play, while longitudinal to transverse coupling can be neglected. ${ }^{1}$

For the membrane, there appears to be no previous study about regimes of oscillations due to the geometric nonlinearity. However, pitch glides due to tension modulation are known to be perceptually also very significant in membranes. An example is the tom-tom drum, where characteristic glides can be heard at medium-high dynamic ranges. ${ }^{5}$ Therefore, there is a strong motivation to simulate tension modulation in physics-based synthesis.

The nonlinear motion of a tension-modulated string was already investigated by Kirchhoff, then revisited by Carrier. ${ }^{6}$ All later tension modulated models proposed in the fields of musical acoustics and sound synthesis are based on the Kirchhoff-Carrier approach.

In the context of physically based sound synthesis, the first study on tension modulation is by Karjalainen et al., ${ }^{7}$ who developed a nonlinear string model for the kantele (a Finnish traditional instrument), using digital waveguides. ${ }^{8}$ Since then, various approaches have been presented in the framework of waveguide modeling: In general, the effect of tension variation can be simulated by varying the delay line 
TABLE I. Main features of the different regimes of string behavior The $\times$ sign means that the specific feature of vibration is significant.

\begin{tabular}{lccc}
\hline \hline Regime & $\mathrm{T} \rightarrow \mathrm{L}$ & $\mathrm{L} \rightarrow \mathrm{T}$ & L inertial eff. \\
\hline Linear motion & & & \\
Double freq. terms & $\times$ & $\times$ & \\
Tension modulation & $\times$ & & $\times$ \\
Longotudinal modes & $\times$ & $\times$ & $\times$ \\
Bidirectional coupling & $\times$ & & \\
\hline \hline
\end{tabular}

length, which can be achieved through a variable allpass filter at the termination. ${ }^{4,9}$ The string tension is estimated at each time instant, then the instantaneous propagation speed and the required length change in the delay line are obtained. The calculation of the tension is demanding and increases the computational load significantly compared with linear string models. The computational complexity is further increased if the variable length delays are distributed between the delay elements for better accuracy. ${ }^{10,11}$

String models based on modal synthesis ${ }^{12}$ are also widely used. In this context, a tension-modulated string model has been proposed by Rabenstein and Trautmann ${ }^{13}$ that is based on the so-called functional transformation method $(\mathrm{FTM})^{14}$ to derive modal parameters. Bilbao also proposed a modal-type approach, ${ }^{15}$ which, unlike other modal synthesis realizations, has an energy-conservation property by which the stability of the numerical model is guaranteed by construction. In finite-difference models, it is relatively straightforward to simulate tension modulation by changing the tension parameter of a linear string model. An energy-conserving scheme was presented by Bilbao. ${ }^{16}$

Compared with strings, tension modulation in membranes is discussed in a significantly smaller amount of literature. Early linear membrane models were mainly based on digital waveguide meshes, ${ }^{17}$ which can provide accurate simulation of wave propagation, depending on mesh topology ${ }^{18}$ and methods to compensate for dispersion. ${ }^{19}$ However, nonlinear models accounting for tension modulation were developed mostly in the context of modal synthesis. Rabenstein and coworkers proposed an extension of their FTM approach to the 2-D case of tension-modulated rectangular membranes. ${ }^{20}$

More general theories for large amplitude vibrations of membranes are typically derived from theories for thin plates subjected to lateral and in-plane forces. ${ }^{21,22}$ One of the most widely used theories was developed by von Karman. ${ }^{23}$ In this approach, plate vibrations are modeled by two nonlinearly coupled PDEs, one for transverse displacement and one for a stress function. The von Karman equations have been applied to the sound synthesis of plates. ${ }^{24,25}$ More recently, a tension-modulated circular membrane model was proposed $^{26,27}$ based on the so-called Berger approximation of the von Karman equations. ${ }^{23,28}$ A finite-difference tension-modulated membrane model has also been recently proposed. $^{29}$

Even for the simplest models of tension modulation in strings and membranes, the computational complexity is significantly higher than for efficient linear models. This paper presents a novel approach, which leads to a simplified description and to significant computational savings in numerical simulations, while still allowing simulation of the perceptually most relevant effect of tension modulation. It is shown that the so-called "quasistatic" component (i.e., the short-time average) of the nonlinear tension, which is responsible for the pitch glide effect, can be estimated from the total energy of the system. Moreover, the system energy can be computed at significantly lower computational costs than the tension. As such, the proposed approach is particularly relevant for applications in the context of physically based sound synthesis of musical instruments and makes it possible to include tension modulation in string and membrane synthesis in less powerful computational environments.

Preliminary results have been already presented for the the string ${ }^{30}$ and the circular membrane. ${ }^{31}$ This paper extends these results in several respects. First, a general formulation is proposed that specializes to the 1-D case of the string and to the 2-D case of the membrane with circular and rectangular geometries. While the methodology can be used for several types of excitation, here it is demonstrated for the case of hammer impact. For this case, a further simplified approach to energy computation is presented that computes the quasistatic component of tension with a negligible computational overhead compared with linear models. The results are demonstrated by numerical simulations of string and membrane vibrations, using both modal synthesis (for the membranes) and finite-difference approaches (for the string).

The remainder of the paper is organized as follows. Section II describes the general initial and boundary value problem and shows that it includes the Kirchhoff-Carrier equation and the Berger equations as special cases. The modal solution is presented in Sec. III, which also shows that the spatially uniform tension can be written in terms of the normal modes. Section IV illustrates the main ideas of the proposed approach and relates the total energy of the system to the tension. Finally, Sec. V applies these results to the efficient synthesis of tension-modulated strings and membranes.

\section{SPATIALLY UNIFORM TENSION MODULATION IN STRINGS AND MEMBRANES}

\section{A. General formulation}

Throughout the paper, the following equation of motion is considered for the displacement $z(\boldsymbol{x}, t)$ defined over the $d$-dimensional domain $\mathcal{S} \subset \mathbb{R}^{d}(d=1,2)$ :

$$
D \nabla^{4} z+\rho \frac{\partial^{2} z}{\partial t^{2}}-T(z) \nabla^{2} z+d_{1} \frac{\partial z}{\partial t}-d_{3} \frac{\partial \nabla^{2} z}{\partial t}=f^{(\mathrm{ext})}
$$

where spatial and temporal dependencies $(x, t)$ (with $x \in \mathcal{S}$ and $t \in \mathbb{R}$ ) have been omitted for brevity. The term $f^{(\mathrm{ext})}(\boldsymbol{x}, t)$ on the right-hand side is an external force density (in $\mathrm{N} / \mathrm{m}^{d}$ ) acting on the system. The operators $\nabla^{2}$ and $\nabla^{4}=\nabla^{2} \cdot \nabla^{2}$ are the $d$-dimensional Laplacian and biharmonic operator, respectively. The constant $\rho$ is the material density (in $\mathrm{kg} / \mathrm{m}^{d}$ ), while the function $T(z)$ represents tension 
(in $\mathrm{N} / \mathrm{m}^{d-1}$ ). We model all types of losses (internal and air losses, losses at the boundary) by the terms with first-order time derivatives and by the $d_{1}, d_{3}$ coefficients. The fourthorder term accounts for the effect of the bending stiffness $D$, which is proportional to the Young's modulus of the material (the constant of proportionality depends on the dimensionality and geometry of the problem). Bending stiffness is assumed to be small $(D / T \ll 1)$ throughout the paper.

Note that Eq. (1) considers the case of spatially uniform tension, i.e., $T$ does not depend on the point $\boldsymbol{x}$, but is a function of the overall displacement function $z$ only. The function $T(z)$ is assumed to have the following form:

$$
T(z)=T_{0}+T_{N L}(z),
$$

where $T_{0}$ is the tension at rest, while $T_{N L}$ depends nonlinearly on the displacement. The following form is considered for $T_{N L}$ :

$$
T_{N L}(z)=C_{N L} \frac{S(z)-S_{0}}{S_{0}} \simeq \frac{1}{2} \frac{C_{N L}}{S_{0}} \int_{\mathcal{S}}\|\nabla z\|^{2} d x,
$$

where $S(z)$ represents the "extension" (e.g., the string length or the membrane area) spanned by the displacement $z$ over the set $\mathcal{S}$, and $S_{0}=\left.S(z)\right|_{z=0}$ is the extension at rest. The general assumption behind Eq. (3) is that tension modulation only depends on the total variation of the extension $S(z)$. The integral in Eq. (3) approximates the variation of $S(z)$. The symbol $\nabla$ indicates gradient, while the constant $C_{N L}$ depends on the problem dimensionality and geometry. The meaning of Eq. (3) is further clarified through the special cases discussed next.

\section{B. Special cases}

The preceding general formulation includes two relevant special cases: the Kirchhoff-Carrier equation ${ }^{6}$ for the tension-modulated string, and the Berger equations for the tension-modulated membrane. ${ }^{23}$

Equation (1) with $d=1$ describes the displacement $z(x, t)$ of a string, where $S=\left\{x: x \in\left[0, L_{0}\right]\right\}$ and $L_{0}$ is the string length at rest. In this case, $\rho$ (in $\mathrm{kg} / \mathrm{m}$ ) is the string linear density and $D=Q a \kappa^{2}$ is the bending stiffness (with $Q$, Young modulus; $a$, cross-sectional string area; $\kappa$, radius of gyration). According to the Kirchhoff-Carrier equation, spatially uniform tension of the string at large displacements is approximated by

$$
T_{N L}(z) \simeq \frac{1}{2} \frac{Q a}{L_{0}} \int_{0}^{L_{0}}\left(\frac{d z}{d x}\right)^{2} d x .
$$

The integral represents the increase of string length at large displacements, $L(z)$, with respect to the length at rest $L_{0}$. This is a special case of Eq. (3) with $C_{N L}=Q a$, and with the string length $L(z)$ having the role of the extension $S(z)$ in the general formulation:

$$
T_{N L}(z)=Q a \frac{L(z)-L_{0}}{L_{0}} .
$$

J. Acoust. Soc. Am., Vol. 131, No. 1, Pt. 2, January 2012
Equation (1) with $d=2$ describes the displacement $z(x, t)$ of a membrane. For a rectangular membrane, $\mathcal{S}=\{\boldsymbol{x}=(x, y)$ : $\left.x \in\left[0, L_{x}\right], y \in\left[0, L_{y}\right]\right\}$, where $L_{x}$ and $L_{y}$ are the membrane lengths at rest in the $x$ and $y$ directions, respectively. For a circular membrane, $\mathcal{S}=\{\boldsymbol{x}=(r, \varphi): r \in[0, R], \varphi \in[0,2 \pi)\}$, where $R$ is the membrane radius. In both cases, $\rho$ (in $\mathrm{kg} / \mathrm{m}^{2}$ ) is the membrane surface density and $D=Q h^{3} / 12\left(1-\nu^{2}\right)$ is the bending stiffness (where $Q$ is the Young modulus, $h$ is the membrane height, and $\nu$ is the Poisson ratio).

A model of spatially uniform tension in a membrane at large displacement is provided by the Berger approximation of von Karman theory. ${ }^{23,28}$ For the rectangular membrane, the Berger approximation can be restated as:

$$
\begin{aligned}
T_{N L}(z) \simeq & \frac{Q h}{2 L_{x} L_{y}\left(1-\nu^{2}\right)} \\
& \cdot \int_{0}^{L_{x}} \int_{0}^{L_{y}}\left[\left(\frac{\partial z}{\partial x}\right)^{2}+\left(\frac{\partial z}{\partial y}\right)^{2}\right] d x d y .
\end{aligned}
$$

For the circular membrane, the equation becomes:

$$
\begin{aligned}
T_{N L}(z) \simeq & \frac{Q h}{2 \pi R^{2}\left(1-v^{2}\right)} \\
& \cdot \int_{0}^{R} \int_{0}^{2 \pi}\left[\left(\frac{\partial z}{\partial r}\right)^{2}+\frac{1}{r^{2}}\left(\frac{\partial z}{\partial \varphi}\right)^{2}\right] r d \varphi d r .
\end{aligned}
$$

These equations can be interpreted as the 2-D version of Eq. (4) because the double integrals represent the increase in membrane area at large displacements, $A(z)$, with respect to the area at rest $A_{0}{ }^{26}$ Thus Eqs. (6) and (7) are special cases of Eq. (3) with $C_{N L}=Q h /\left(1-v^{2}\right)$, and with the membrane area $A(z)$ having the role of the extension $S(z)$ in the general formulation:

$$
T_{N L}(z)=\frac{Q h}{1-v^{2}} \frac{A(z)-A_{0}}{A_{0}},
$$

independently of the membrane geometry.

Figure 1 exemplifies the effects of spatially uniform tension modulation in two cases. Figure 1(a) shows a synthetic example of a rigidly terminated, tension-modulated string, obtained from a finite-difference model. The pluck exciting the string is modeled as a triangle-shaped initial displacement distribution with a peak value of $5 \mathrm{~mm}$. The finitedifference model is a variation of the linear model of Chaigne and Askenfelt, ${ }^{32}$ in which the tension parameter varies according to the tension computed from the string displacement by the discretized version of Eq. (4). Figure 1(b) shows a synthetic example of a tension-modulated circular membrane, obtained using modal synthesis and an impact force model that simulates interaction with a hammer or mallet $^{26}$ with impact velocity $10 \mathrm{~m} / \mathrm{s}$, that is well below the highest dynamic levels in drum playing. ${ }^{33}$

For the string example, the tension at rest is $T_{0}=100 \mathrm{~N}$, whereas for the membrane, it is $T_{0}=1500 \mathrm{~N} / \mathrm{m}$. Therefore in both cases, the magnitude of $T_{N L}$ is a significant fraction of $T_{0}$ and causes audible pitch glides. In the same figures, the dashed lines show the short-time average (quasistatic) 


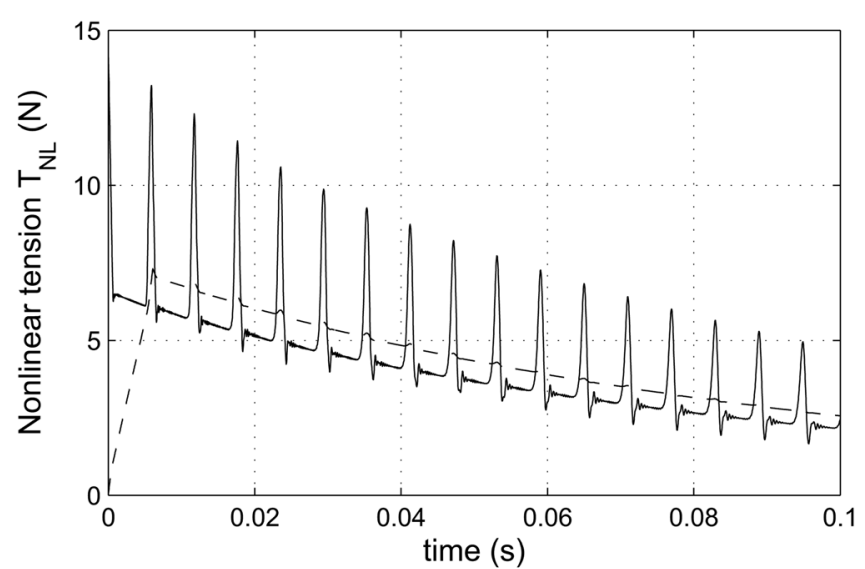

(a)

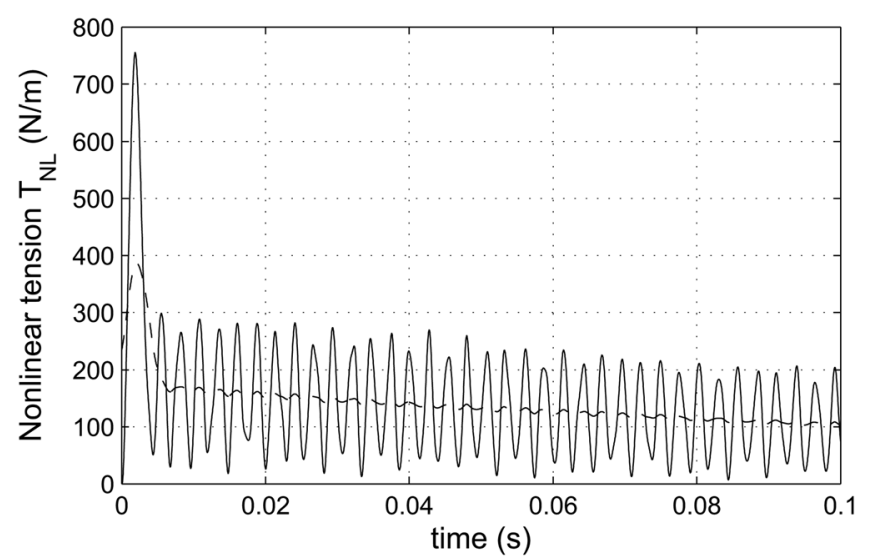

(b)

FIG. 1. Simulated nonlinear tension $T_{N L}$ (solid) and quasistatic tension component (dashed) in two cases: (a) electric guitar string with $L_{0}=65 \mathrm{~cm}$, plucked at point $0.12 L_{0}$; (b) circular membrane with $R=16 \mathrm{~cm}$, hit with velocity $10 \mathrm{~m} / \mathrm{s}$ at point $0.5 R$.

tension variation. The slow initial rise of this tension component occurs because this has been estimated by applying a running average filter to $T_{N L}$.

\section{MODAL SOLUTION}

\section{A. General formulation}

Equation (1) has a unique solution for given boundary and initial conditions. Here we consider the case of zero displacement and zero second-order spatial derivative on the boundary. The latter condition is needed if the fourth-order term is considered in Eq. (1).

Let us first consider Eq. (1) in the linear case $\left(T_{N L}(z) \equiv 0\right)$. Recall that a mode is a particular solution in which temporal and spatial dependencies are decoupled. With the considered boundary conditions, Eq. (1) has a numerable set of modes

$$
\bar{z}_{\eta}(t) K_{\eta}(\boldsymbol{x}), \eta \in \mathbb{N}^{d}
$$

where $d$ is the dimension of the problem. In the hypothesis of vanishingly small bending stiffness and dissipation, the space-dependent functions $K_{\eta}$ (i.e., the modal shapes) are the eigenfunctions of the operator $\nabla^{2}$ satisfying the boundary conditions of the problem:

$$
\nabla^{2} K_{\eta}(\boldsymbol{x})=-\lambda_{\eta} K_{\eta}(\boldsymbol{x})
$$

where $-\lambda_{\eta}$ (with $\lambda_{\eta}>0$ ) are the corresponding eigenvalues. Therefore the functions $K_{\eta}$ depend on the dimensionality and on the geometry of the problem.

The modal solution is associated with a Sturm-Liouville (SL) transform, an integral operator whose kernel is given by the spatial eigenfunctions. ${ }^{14}$ The SL transform $\bar{z}$ of $z$ and the inverse SL transform are defined as

$$
\begin{aligned}
& \bar{z}_{\eta}(t)=\int_{\mathcal{S}} z(\boldsymbol{x}, t) K_{\eta}(\boldsymbol{x}) d \boldsymbol{x}, \\
& z(\boldsymbol{x}, t)=\sum_{\eta} \frac{\bar{z}_{\eta}(t) K_{\eta}(\boldsymbol{x})}{\left\|K_{\eta}(\boldsymbol{x})\right\|_{2}^{2}},
\end{aligned}
$$

where $\|\cdot\|_{2}$ is the norm in $\mathrm{L}^{2}(\mathcal{S})$. Equation (11b) expresses the displacement $z(\boldsymbol{x}, t)$ as the series of its normal modes, with shapes $K_{\eta}(\boldsymbol{x})$ and amplitudes $\bar{z}_{\eta}(t)$.

By substituting Eq. (11b) into the linear equation of motion Eq. (1) and applying Eq. (11a), one obtains a set of second-order ordinary differential equations that describe the dynamics of the normal modes:

$$
\ddot{\bar{z}}_{\eta}+\frac{d_{1}+d_{3} \lambda_{\eta}}{\rho} \dot{\bar{z}}_{\eta}+\lambda_{\eta}\left(\lambda_{\eta} \frac{D}{\rho}+c^{2}\right) \bar{z}_{\eta}=\frac{\bar{f}_{\eta}^{(\mathrm{ext})}}{\rho},
$$

where

$$
\bar{f}_{\eta}^{(\mathrm{ext})}=\int_{\mathcal{S}} f^{(\mathrm{ext})}(\boldsymbol{x}, t) K_{\eta}(\boldsymbol{x}) d \boldsymbol{x}
$$

is the excitation force acting on mode $\eta$, and $c=\sqrt{T_{0} / \rho}$ is the propagation speed. The solutions for the homogenous part $\left(\bar{f}_{\eta}^{\text {(ext })}=0\right)$ of Eq. (12) are exponentially decaying sinusoids:

$$
\bar{z}_{\eta}(t)=A_{\eta} \sin \left(\omega_{\eta} t+\phi_{\eta}\right) e^{-t / \tau \eta}
$$

where amplitudes $A_{\eta}$ and phases $\phi_{\eta}$ depend on the initial conditions. The modal frequencies $\omega_{\eta}$ and decay times $\tau_{\eta}$ depend on the parameters in Eq. (12):

$$
\begin{aligned}
& \omega_{\eta}^{2}=\lambda_{\eta}\left(\frac{D}{\rho} \lambda_{\eta}+c^{2}\right) \approx \lambda_{\eta} c^{2}, \\
& \tau_{\eta}^{-1}=\frac{1}{2 \rho}\left(d_{1}+d_{3} \lambda_{\eta}\right),
\end{aligned}
$$

where Eq. (15a) holds because $D / T_{0}=D /\left(\rho c^{2}\right) \ll 1$.

Let us now take into account the nonlinear tension. The term involving $T_{N L}$ can be moved to the right-hand side of Eq. (1) and regarded as an additional force $f^{(\mathrm{tm})}(z)=$ $T_{N L}(z) \nabla^{2} z$ acting on the system. Applying the SL transform to $f^{(\mathrm{tm})}$ yields, for the $\eta$ th mode: 


$$
\bar{f}_{\eta}^{(\mathrm{tm})}(\bar{z}, z)=\int_{\mathcal{S}}\left[T_{N L}(z) \nabla^{2} z\right] K_{\eta} d \boldsymbol{x}=-\lambda_{\eta} T_{N L}(z) \bar{z}_{\eta} .
$$

This tension modulation force can be incorporated into Eq. (12) in a similar way to $\bar{f}_{\eta}^{(\text {ext })}$. By substituting Eq. (11b) into Eq. (3), and exploiting mode orthogonality, $T_{N L}$ in Eq. (3) is written as a function of the modes:

$$
\bar{T}_{N L}(\bar{z})=\frac{1}{2} \frac{C_{N L}}{S_{0}} \sum_{\eta} \frac{\lambda_{\eta} \bar{z}_{\eta}^{2}(t)}{\left\|K_{\eta}\right\|_{2}^{2}} .
$$

Therefore $\bar{f}_{\eta}^{(\mathrm{tm})}$ can be written as

$$
\bar{f}_{\eta}^{(\mathrm{tm})}(\bar{z})=-\lambda_{\eta} \frac{1}{2} \frac{C_{N L}}{S_{0}}\left[\sum_{\bar{\eta}} \frac{\lambda_{\bar{\eta}} \bar{z}_{\eta}^{2}(t)}{\left\|K_{\bar{\eta}}\right\|_{2}^{2}}\right] \bar{z}_{\eta} .
$$

\section{B. Special cases}

Again the preceding general formulation can be specialized to the relevant cases under examination, i.e., the tension modulated string and membrane with fixed boundary conditions. Modal solutions for these systems are known and are summarized next.

For the string, spatial eigenfunctions are: ${ }^{34}$

$$
K_{n}(x)=\sin \left(\frac{n \pi x}{L_{0}}\right)
$$

where $n=0, \ldots,+\infty$. These satisfy

$$
\nabla^{2} K_{n}(x)=-\lambda_{n} K_{n}(x), \text { with } \lambda_{n}=\left(\frac{n \pi}{L_{0}}\right)^{2} .
$$

For the rectangular membrane, the spatial eigenfunctions are: ${ }^{35}$

$$
K_{n, m}(x, y)=\sin \left(\frac{n \pi x}{L_{x}}\right) \sin \left(\frac{m \pi y}{L_{y}}\right)
$$

where $n, m=0, \ldots,+\infty$. These satisfy

$$
\begin{aligned}
& \nabla^{2} K_{n, m}(x, y)=-\lambda_{n, m} K_{n, m}(x, y), \\
& \text { with } \quad \lambda_{n, m}=\pi^{2}\left[\left(\frac{n}{L_{x}}\right)^{2}+\left(\frac{m}{L_{y}}\right)^{2}\right] .
\end{aligned}
$$

For the circular membrane, the spatial eigenfunctions are ${ }^{35}$ :

$$
K_{n, m}(r, \varphi)=\cos (n \varphi) J_{n}\left(\mu_{n, m} \frac{r}{R}\right)
$$

where $n=0, \ldots,+\infty, m=1, \ldots,+\infty$, and $\mu_{n, m}$ is the $m$ th zero of the $n$th order Bessel function of the first kind, $J_{n}$. These satisfy

$$
\nabla^{2} K_{n, m}(x)=-\lambda_{n, m} K_{n, m}(x), \text { with } \quad \lambda_{n, m}=\left(\frac{\mu_{n, m}}{R}\right)^{2}
$$

Equations (19), (21), and (23) are all special cases of the general modal solution, with $\eta=n$ for the string and $\eta=(n, m)$ for the membrane.

\section{ENERGY-BASED MODEL OF TENSION MODULATION}

Because the most prominent perceptual effect of tension modulation in strings and membranes is the pitch glide due to the quasistatic variation of the tension with oscillation amplitude, it is reasonable to focus on the modeling of this effect only. In this section, we first show that the quasistatic tension component is responsible for the pitch glide effect and then derive a simple relationship between this component and the energy of the system. As will be shown later, the energy of the system can be estimated at lower computational complexity, thus allowing computation of the quasistatic tension with less operations than earlier tension modulation models.

\section{A. Quasistatic tension modulation}

The nonlinear tension can be split into a quasistatic component and a second one containing double-frequency terms. By substituting Eq. (14) into Eq. (17), one finds:

$$
\bar{T}_{N L}(\bar{z})=\frac{C_{N L}}{2 S_{0}} \sum_{\eta} \frac{\lambda_{\eta} A_{\eta}^{2}}{\left\|K_{\eta}\right\|_{2}^{2}}\left[1-\cos \left(2 \omega_{\eta} t+2 \phi_{\eta}\right)\right] e^{-2 t / \tau_{\eta}}
$$

The first time-dependent part of Eq. (25) is a quasistatic variation of tension:

$$
T_{q s}(t)=\frac{1}{2} \frac{C_{N L}}{S_{0}} \sum_{\eta} \frac{\lambda_{\eta} A_{\eta}^{2}}{\left\|K_{\eta}\right\|_{2}^{2}} \cdot e^{-2 t / \tau_{\eta}}
$$

which decays slowly with respect to the oscillation periods $2 \pi / \omega_{\eta}$. This leads to a shift (continuous decrease) in the modal frequencies, resulting in a pitch glide. Figures 1(a) and 1(b) show estimates of $T_{q s}(t)$ for the plucked string and the struck membrane obtained by applying a lowpass filter to the respective $T_{N L}$ signals.

The second part of Eq. (25) contains the double frequency terms

$$
T_{d f}(t)=-\frac{C_{N L}}{2 S_{0}} \sum_{\eta} \frac{\lambda_{\eta} A_{\eta}^{2}}{\left\|K_{\eta}\right\|_{2}^{2}} \cos \left(2 \omega_{\eta} t+2 \phi_{\eta}\right) e^{-2 t / \tau_{\eta}}
$$

and produces a continuous modulation of tension, built up by sinusoidal functions with doubled oscillation frequencies compared with the corresponding modal frequencies. The amplitude of this modulation decays exponentially, and the decay times of its components are halved with respect to the modal ones.

For the string, the effect of the double frequency terms has been discussed in the literature. ${ }^{36}$ If the string is rigidly terminated, transverse modes cannot efficiently exchange energy and double frequency terms do not have an effect in practice, and only the quasi-static part is relevant. If the bridge is not infinitely rigid (so that the modal shapes are not anymore orthogonal), all the modes can gain energy from the bridge motion. However, this mode coupling effect is not significant for most western string instruments due to the 
large difference in the order of magnitude of string and bridge admittances.

For the membrane, the effect of double frequency terms has not been discussed in the literature to our knowledge and is an interesting topic for future research. We assume that the various modes might gain energy from each other even for rigid terminations due to the much higher modal density than that of the string. Nevertheless, modeling only the quasistatic part still captures the most relevant perceptual features of tension modulation as will be demonstrated by the examples in Sec. V.

\section{B. Energy}

The following derivations can be found in acoustics textbooks $^{37}$ and are summarized here to make the paper self-explanatory. The kinetic energy of an element $d \boldsymbol{x}$ is $d E_{k}(\boldsymbol{x}, t)=1 / 2 \rho d \boldsymbol{x} \dot{z}^{2}(\boldsymbol{x}, t)$. The total kinetic energy is

$$
E_{k}(t)=\frac{\rho}{2} \int_{\mathcal{S}}\left[\frac{\partial z(\boldsymbol{x}, t)}{\partial t}\right]^{2} d \boldsymbol{x} .
$$

The potential energy $E_{p}$ can be derived as follows. Consider a path that goes from equilibrium to the final displacement $z$, through the intermediate displacements $k z$ (with $k \in[0,1]$ ). The potential energy equals the work done by the system forces along this path. Throughout the path, the force acting on an element $d \boldsymbol{x}$ is

$$
F(\boldsymbol{x}, k)=T_{0} \nabla^{2} k z(\boldsymbol{x}, t) d \boldsymbol{x}
$$

At the final displacement $z$, the potential energy $d E_{p}$ of the element $d \boldsymbol{x}$ equals (with opposite sign) the work done by this force along the whole path. For each increase $d k$, the corresponding change in displacement is $z d k$, thus:

$$
\begin{aligned}
d E_{p}(\boldsymbol{x}, t) & =-\int_{0}^{1}\left[T_{0} \nabla^{2} k z(\boldsymbol{x}, t) d \boldsymbol{x}\right] z(\boldsymbol{x}, t) d k \\
& =-\frac{T_{0}}{2} \nabla^{2} z(\boldsymbol{x}, t) z(\boldsymbol{x}, t) d \boldsymbol{x} .
\end{aligned}
$$

Therefore, the total potential energy is

$$
E_{p}(t)=-\frac{T_{0}}{2} \int_{\mathcal{S}} \nabla^{2} z(\boldsymbol{x}, t) z(\boldsymbol{x}, t) d \boldsymbol{x} .
$$

We have implicitly assumed $E_{p}=0$ at equilibrium. Note also that Eq. (31) represents in fact $E_{p}$ in the linear case, as the forces acting on $d \boldsymbol{x}$ are estimated in Eq. (29) by considering $T_{0}$ only and discarding $T_{N L}$. This approximation permits to derive a simple relation between $T_{q s}$ and the total system energy $E=E_{k}+E_{p}$ as will be shown next. Moreover, simulation results confirm that the error introduced by this approximation is small.

\section{Quasistatic tension and total energy}

We are now able to show that the quasistatic tension component $T_{q s}$ is directly proportional to the system energy.
This can be proved by rewriting the energy as a function of the modes.

Regarding the kinetic energy $E_{k}$, substitution of Eq. (11b) into Eq. (28) yields

$$
E_{k}(t)=\frac{\rho}{2} \sum_{\eta} \frac{\dot{z}_{\eta}^{2}(t)}{\left\|K_{\eta}\right\|_{2}^{4}} \int_{\mathcal{S}} K_{\eta}^{2}(\boldsymbol{x}) d \boldsymbol{x}=\frac{\rho}{2} \sum_{\eta} \frac{\dot{z}_{\eta}^{2}(t)}{\left\|K_{\eta}\right\|_{2}^{2}} .
$$

The potential energy $E_{p}$ can be rewritten in terms of the modes by substituting Eq. (11b) into Eq. (31). Recalling that the shapes $K_{\eta}$ are orthogonal eigenfunctions of $\nabla^{2}$ with eigenvalues $-\lambda_{\eta}$, one finds

$$
\begin{aligned}
E_{p}(t) & =-\frac{T_{0}}{2} \int_{\mathcal{S}}\left[\sum_{\eta}-\frac{\lambda_{\eta} z_{\eta}^{2}(t)}{\left\|K_{\eta}\right\|_{2}^{4}} K_{\eta}^{2}(\boldsymbol{x})\right] d \boldsymbol{x} \\
& =\frac{T_{0}}{2} \sum_{\eta} \frac{\lambda_{\eta}}{\left\|K_{\eta}\right\|_{2}^{2}} z_{\eta}^{2}(t) .
\end{aligned}
$$

Therefore the total energy is

$$
E=E_{k}+E_{p}=\frac{1}{2} \sum_{\eta} \frac{1}{\left\|K_{\eta}\right\|_{2}^{2}}\left[\rho \dot{\bar{z}}_{\eta}^{2}+T_{0} \lambda_{\eta} \bar{z}_{\eta}^{2}\right] .
$$

Substituting Eq. (14) into this equation, and using the approximation in Eq. (15a), finally leads to

$$
E(t)=\frac{T_{0}}{2} \sum_{\eta} \frac{\lambda_{\eta} A_{\eta}^{2}}{\left\|K_{\eta}\right\|_{2}^{2}} \cdot e^{-2 t / \tau_{\eta}}
$$

Comparison of this equation with Eq. (26) proves that

$$
T_{q s}(t)=\frac{C_{N L}}{S_{0} T_{0}} E(t)
$$

which is the fundamental outcome of this section.

Figure 2 shows a synthetic example of a tensionmodulated rectangular membrane, obtained from the same model of Fig. 1(b). The energies, computed from Eqs. (28) and (31), are plotted in Fig. 2(a). The energy-based estimation of $T_{q s}$ is plotted in Fig. 2(b), along with the nonlinear tension $T_{N L}$. As a term of comparison, the figure also plots an estimate of $T_{q s}$ [dotted line in Fig. 2(b)] obtained by applying a lowpass filter to $T_{N L}$.

The kinetic and potential energies oscillate in antiphase, and the total energy is a slowly decaying signal as expected. Note that the total energy is not exactly a monotonically decreasing signal and exhibits small oscillations instead. This is due to the fact that the potential energy $E_{p}$ in Eq. (31) has been derived in the linear regime, discarding the effects of tension modulation.

The example shows that the quasistatic tension can be accurately computed from the energy. Similar plots are obtained for the string and the circular membrane.

\section{EFFICIENT SOUND SYNTHESIS}

As already mentioned, computing the nonlinear tension component $T_{N L}$ at sample rate is an expensive operation. As 


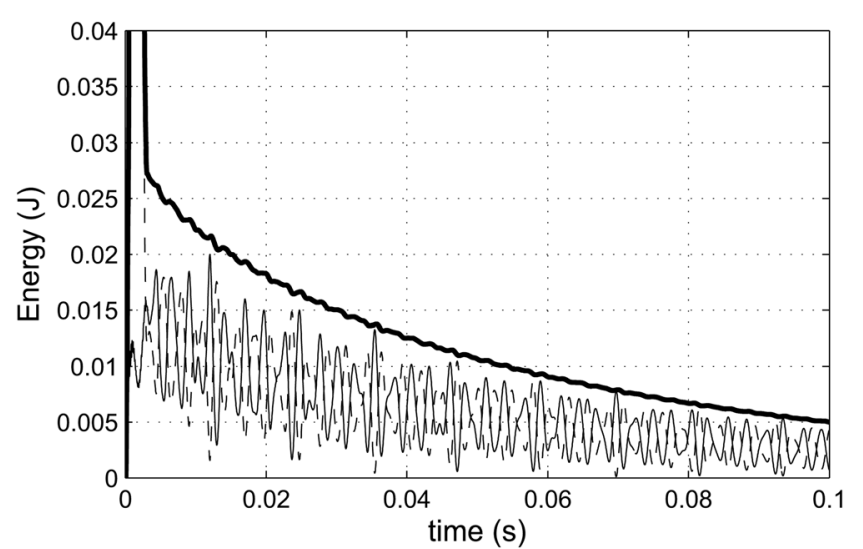

(a)

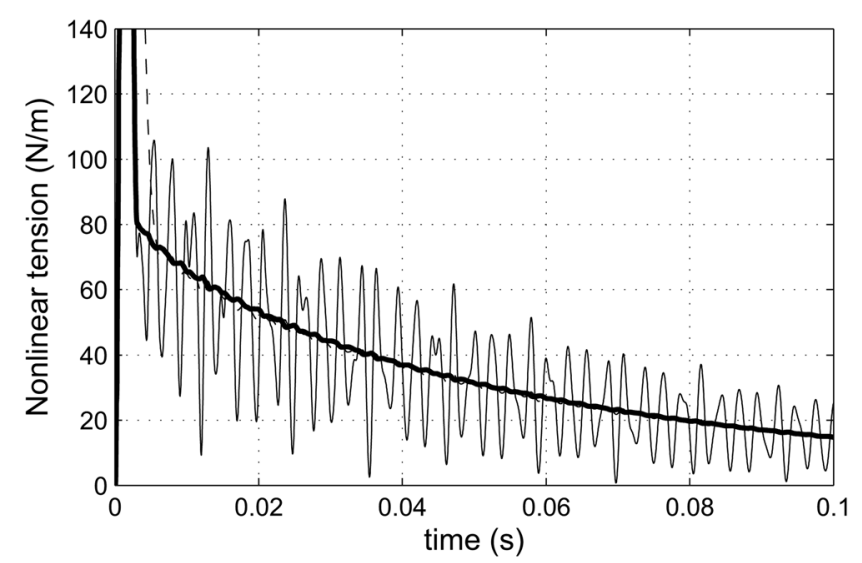

(b)

FIG. 2. Simulated rectangular membrane with $\left(L_{x}, L_{y}\right)=(32,25) \mathrm{cm}$ and $T_{0}=1500 \mathrm{~N} / \mathrm{m}$, hit with velocity $10 \mathrm{~m} / \mathrm{s}$ at point $\left(0.3 L_{x}, 0.1 L_{y}\right)$; (a) total (solid thick), kinetic (solid), and potential (dashed) energies; (b) nonlinear tension $T_{N L}$ (solid), quasistatic tension $T_{q s}$ computed from energy (solid thick) and estimated by lowpass filtering $T_{N L}$ (dashed).

an example, Eq. (17) requires calculation of a weighted sum of the squared modal displacements (in the order of several hundreds or thousands).

This section shows that the energy-based model introduced in the preceding text can be exploited to develop efficient sound synthesis algorithms simulating tensionmodulated strings and membranes. Two examples are provided. The first one uses a finite-difference string model that extends the model by Chaigne and Askenfelt, ${ }^{32}$ where the tension parameter is not constant but varied according to our energy-based tension model. The second example uses a membrane model based on modal synthesis ${ }^{26}$ in which the time-varying tension modulation term is substituted by the slowly varying energy-based quasi-static tension. The same ideas could also be applied to waveguide models or other sound synthesis paradigms.

\section{A. Downsampled energy computation}

Substituting the computation of tension with the computation of a scaled energy signal will not yet lead to computational savings because energy computation [from Eqs. (28) and (31)] takes a similar number of operations as tension computation. However, the complexity of the energy computation can be decreased significantly.

Because $E(t)$ is a slowly varying signal, in contrast to $T_{N L}(t)$, it can be computed at a lower rate and linearly interpolated between the computed points without significant losses in accuracy. As a result, the average load of energy computation becomes negligible compared with the samplerate computation of the modal displacements.

It has to be noted that such downsampled energy computation introduces a delay that equals the downsampling factor. However, this delay arguably does not affect significantly the accuracy of the simulation, given the slowly varying nature of the energy. This conjecture is confirmed by the simulation results in Sec. V C.

\section{B. Energy storage model}

Here we present a second method for efficient energy computation that requires even less computations than the multirate method above. From Fig. 2 and Eq. (26), one can see that $T_{q s}$ decays almost exponentially. The decay would be exactly exponential if the modal decay times $\tau_{\eta}$ were the same for all $\eta$. In reality, they are different, but usually for the lowest, dominant modes they are in the same order, and the resulting energy and quasistatic tension curves are close to exponential.

Therefore it is appropriate to model the energy storage of the system as a first-order discrete-time lowpass filter the decay time of which is determined by the system dissipation, and where the input and the ouput signals are the amount of energy $\Delta E[n]$ injected into the system during excitation, and the system energy $E[n]$, respectively:

$$
E[n]=\Delta E[n]+g E[n-1]
$$

where $g$ is a constant determining the rate of energy decay. By virtue of the law of conservation of energy, the amount of energy $\Delta E[n]$ injected into the system equals the energy loss of the excitation.

As a simple example, if an initial displacement and/or velocity distribution is given (e.g., a triangle-shaped initial displacement for an ideally plucked string), then $\Delta E[n]=0$, the initial value $E[0]$ is set to the energy of the initial state of the system, and the discrete-time energy $E[n]$ decays exponentially from the initial value.

In a more general case of a dynamic excitation (like a physical model of a pluck or strike), the input $\Delta E[n]$ has to be given. The general idea is illustrated for the case of a hammer strike because striking is one of the most common forms of excitation in string and membrane instruments. A hammer or mallet hitting a string or a membrane can be modeled $^{32,38,39}$ as a point-like mass $m_{\mathrm{h}}$, which generates a nonlinear elastic impact force $F_{\mathrm{h}}$ at the hit point:

$$
F_{h}(\Delta z)= \begin{cases}k_{h}(\Delta z)^{\alpha_{h}} \Delta z>0, \\ 0 & \Delta z \leq 0,\end{cases}
$$

where $\Delta z(t)=z\left(\boldsymbol{x}_{h}, t\right)-z_{h}(t)$ represents hammer compression as the difference between the displacement at the hit 
point $x_{\mathrm{h}}$ and the hammer displacement $z_{\mathrm{h}}, k_{\mathrm{h}}$ is the force stiffness, and the exponent $\alpha_{\mathrm{h}}$ depends on the local geometry around the contact area. The hammer energy during contact is

$$
E_{h}=\frac{1}{2} m_{h} \dot{z}_{h}^{2}+k_{h} \frac{\Delta z^{\alpha_{h}+1}}{\alpha_{h}+1}
$$

and can therefore be computed at every time instant. The energy input to the system is then the energy change of the hammer:

$$
\Delta E[n]=-\left(E_{h}[n]-E_{h}[n-1]\right) .
$$

In summary, the proposed energy storage model realizes a tension-modulated string or membrane model with essentially the same computational load as that of a linear model. The only required additional sample-rate operations are the sums and multiplications needed to

(1) Compute $\Delta E[n]$ by Eqs. (39) and (40) during contact.

(2) Estimate $E[n]$ by the filter of Eq. (37).

(3) Compute $T_{q s}[n]$ by Eq. (36).

For a lossy excitation mechanism, such as a hysteretic hammer, ${ }^{40,41}$ the dissipation of the damping element has to be subtracted from the energy input $\Delta E[n]$.

Figure 3 shows the log energy decay of a circular membrane [from the same model of Fig. 1(b)], struck at a point $r_{h}=0.5 R$ with impact velocity $20 \mathrm{~m} / \mathrm{s}$. An almost linear decay is observed on a logarithmic scale as expected. The dashed line is the energy computed by the energy storage model of Eq. (37) and the hammer energy of Eq. (39). The $g$ parameter of Eq. (37) was estimated by fitting a line on the log energy plot, although it can also be estimated from the decay times of the first modes. The small discrepancy between the actual and modeled energies is most probably due to the fact that modeling the energy loss by Eq. (37) also during excitation is only a rough approximation.

Compared with the downsampled energy computation, the energy storage model leads to lower computational complexity. On the other hand, it requires the formulation of the energy of the exciter. In general, for such excitations where the energy can be expressed in a closed form (like for the

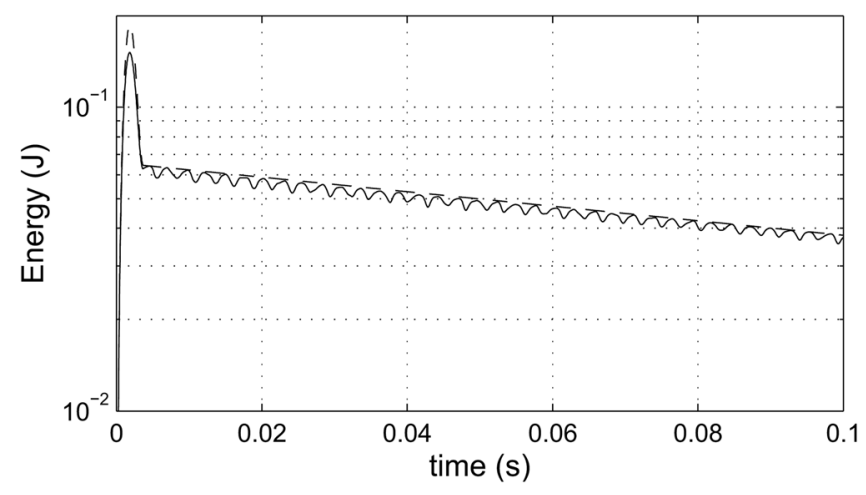

FIG. 3. Log-scale energy decay of a simulated circular membrane excited by an impact force: membrane energy (solid line) and estimated energy computed by the energy storage model of Eq. (37) (dashed line). simple pluck and impact models used in this paper), this latter method should be used because of its computational efficiency.

\section{Results}

Simulation results are summarized in the spectrograms of Figs. 4 and 5. All have been obtained through short-time Fourier transform (STFT) analysis, with a frame length and a hop-size of 8192 and 128 samples, respectively (with sampling rate of $44.1 \mathrm{kHz}$ ). The energy spreading in frequency observed during the attack is the effect of the STFT analysis on the exponentially decaying modal frequencies.

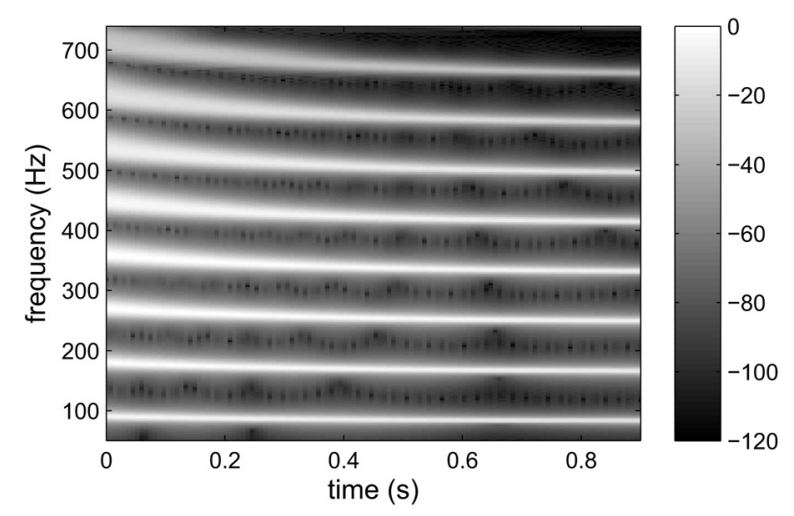

(a)

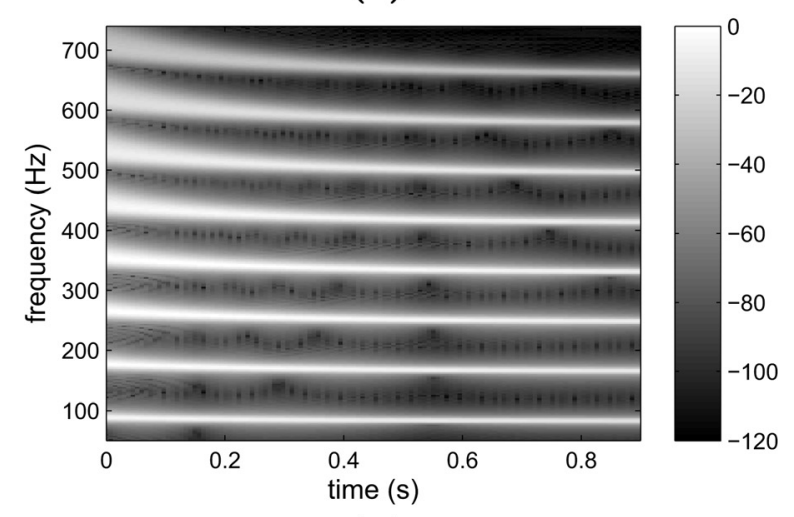

(b)

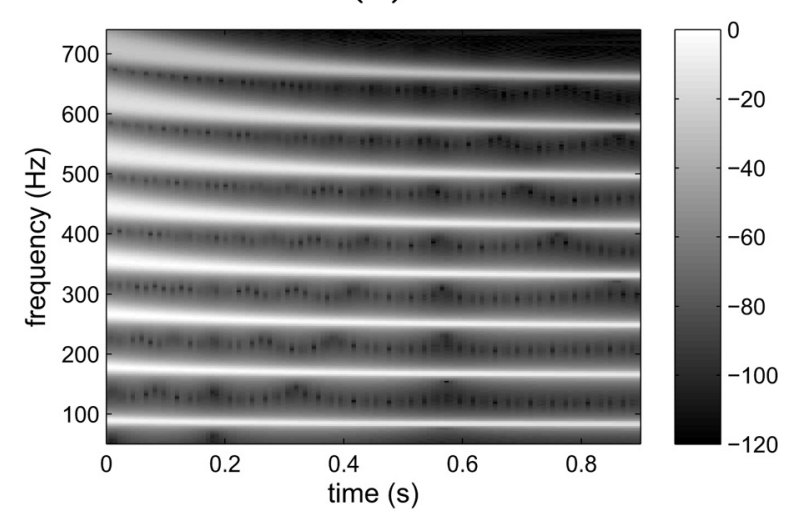

(c)

FIG. 4. Spectrograms (with SPL in dB) of a simulated string; (a) complete model using nonlinear tension $T_{N L}$ of Eq. (17); (b) efficient model using quasistatic tension $T_{q s}$, computed from energy via Eq. (36), with a downsampling factor of 32; (c) efficient model using the energy storage model of Eq. (37) to compute the quasistatic tension $T_{q s}$. 


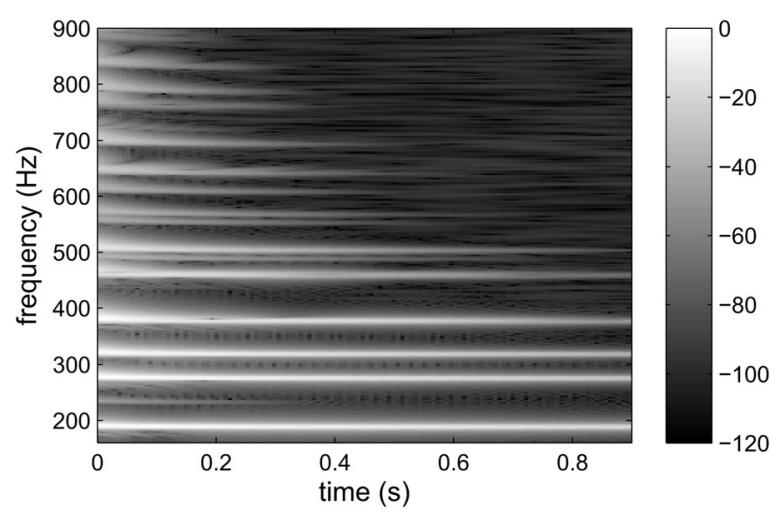

(a)

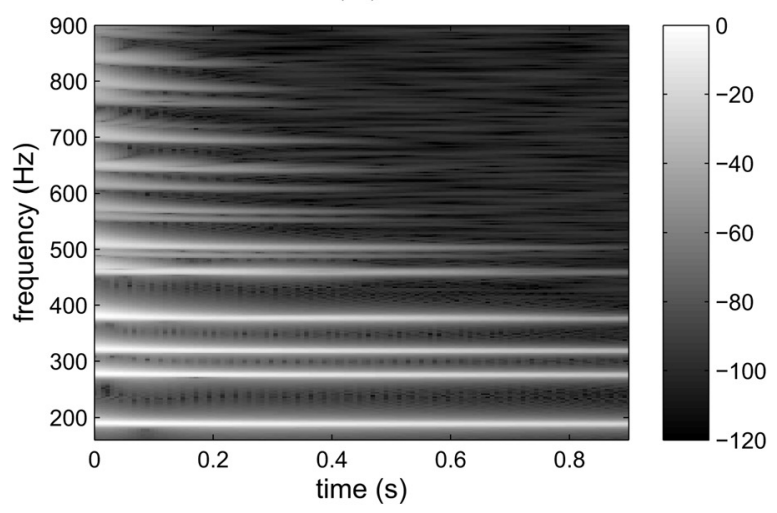

(b)

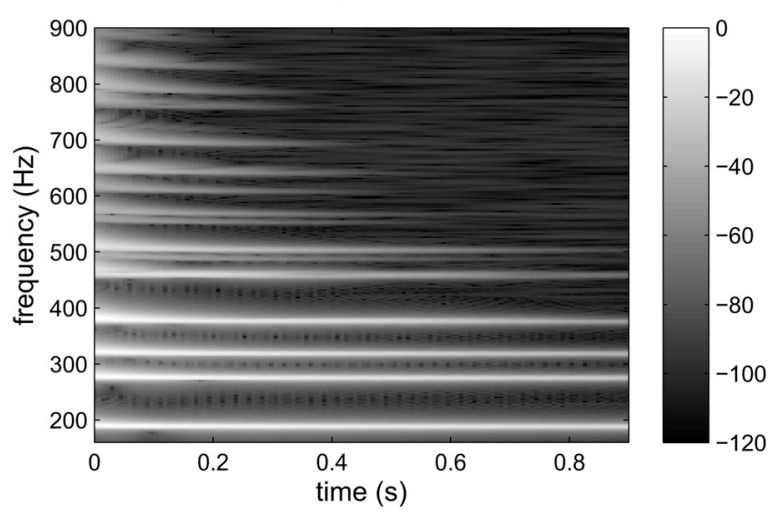

(c)

FIG. 5. Spectrograms (with SPL in $\mathrm{dB}$ ) of a simulated rectangular membrane; (a) complete model using nonlinear tension $T_{N L}$ of Eq. (17); (b) efficient model using quasistatic tension $T_{q s}$, computed from energy via Eq. (36), with a downsampling factor of 32; (c) efficient model using the energy storage model of Eq. (37) to compute the quasistatic tension $T_{q s}$.

The three spectrograms in Fig. 4 are all obtained by plucking the same finite-difference string model of Fig. 1(a). Here the triangle-shaped initial displacement distribution has a peak value of $10 \mathrm{~mm}$. Figure 4(a) corresponds to earlier tension modulation models, where the tension $T_{N L}$ is computed from the string displacement by the discretized version of Eq. (4). The spectrogram in Fig. 4(b) instead has been obtained by approximating $T_{N L}$ with $T_{q s}$, estimated from the string energy according to Eq. (36), and computed with a downsampling factor 32 , i.e., every $32 / 44.1 \sim 0.73 \mathrm{~ms}$. Finally, the spectrogram in Fig. 4(c) has been obtained by approximating the string energy by the energy storage model of Eq. (37) and by computing $T_{q s}$ from this estimated energy.

Figure 5 shows three spectrograms of a rectangular membrane, all obtained by striking the same modal-based membrane model of Fig. 2. Here the impact velocity is 20 $\mathrm{m} / \mathrm{s}$, which is close to the highest dynamic levels in drum playing. 33

According to the spectrograms and informal listening tests, the results of the different models are basically indistinguishable for the rigidly terminated string. This is expected from theory because mode coupling does not occur for rigid terminations and only the quasistatic part of tension variation has an effect, as discussed in Sec. IV A.

This is not exactly the case for the membrane, probably due to the mode coupling that can still arise in the full model even for rigid boundaries (see Sec. IV A). However, the main goal of synthesizing pitch glides correctly is still accomplished. Compared with the full tension modulation model, the efficient models trade a less relevant phenomenon (some mode coupling) for significantly lower computational complexity. Formal listening tests on perceptual differences of the models are out of the scope of this paper and are left for future research. Interested readers may listen to the sound examples at http://www.dei.unipd.it/ avanzini/demos/ jasa2011/.

\section{CONCLUSIONS}

This paper has presented a unified formulation to the tension modulation problem for strings and membranes. For the membrane, circular and rectangular geometries were considered.

By expressing both tension and energy of vibration as functions of modal amplitudes, it was shown that the quasistatic (short-time average) part of tension variation is linearly proportional to the energy of the system. This allowed the development of efficient tension-modulated sound synthesis models that simulate pitch glides in musical instruments at a negligible additional computational cost to that incurred by linear string and membrane models. The efficiency comes from the fact that the system energy is estimated at a significantly lower computational complexity compared with tension calculation.

For this, two methods were proposed. The first one computes energy at a lower sampling rate, and uses linear interpolation in between. The second one models the energy decay by a first order lowpass filter the input of which is the energy loss of the exciter (e.g., the hammer). Both methods allow the accurate synthesis of pitch glides and require lower computational complexity compared with earlier tensionmodulated models.

\section{ACKNOWLEDGMENTS}

The work of Balázs Bank was supported by the EEA and Norway Grants and the Zoltán Magyary Higher Education Foundation. We are thankful to Dr. Stefan Bilbao, Dr. Federico Fontana, and the anonymous reviewers for their helpful comments. 
${ }^{1}$ B. Bank, "Physics-based sound synthesis of string instruments including geometric non-linearities," Ph.D. thesis, Budapest University of Technology and Economics, Budapest, 2006.

${ }^{2}$ S. Bilbao, "Conservative numerical methods for nonlinear strings," J. Acoust. Soc. Am. 118, 3316-3327 (2005).

${ }^{3}$ S. Bilbao, Numerical Sound Synthesis_Finite Difference Schemes and Simulation in Musical Acoustics (Wiley, Chichester, 2009), Chap. 8, pp. 221-247.

${ }^{4}$ C. Erkut, M. Karjalainen, P. Huang, and V. Välimäki, “Acoustical analysis and model-based sound synthesis of the kantele," J. Acoust. Soc. Am. 112, 1681-1691 (2002).

${ }^{5}$ N. H. Fletcher and T. D. Rossing, The Physics of Musical Instruments (Springer-Verlag, New York, 1991), Chap. 18, pp. 516-517.

${ }^{6} \mathrm{G}$. F. Carrier, "On the nonlinear vibrations problem of elastic string," Quart. J. Appl. Math. 3, 157-165 (1945).

${ }^{7}$ M. Karjalainen, J. Backman, and J. Pölkki, "Analysis, modeling, and realtime sound synthesis of the kantele, a traditional finnish string instrument," Proceedings of the IEEE International Conference on Acoustics, Speech and Signal Processing, Minneapolis, MN (1993), Vol. 1, pp. 229-232.

${ }^{8}$ J. O. Smith III, "Principles of digital waveguide models of musical instruments," in Applications of Digital Signal Processing to Audio and Acoustics, edited by M. Kahrs and K.-H. Brandenburg (Kluwer Academic Publishers, New York, 1998), pp. 417-466.

${ }^{9}$ T. Tolonen, V. Välimäki, and M. Karjalainen, "Modeling of tension modulation nonlinearity in plucked strings," IEEE Trans. Speech Audio Process. 8, 300-310 (2000).

${ }^{10}$ J. Pakarinen, V. Välimäki, and M. Karjalainen, "Physics-based methods for modeling nonlinear vibrating strings," Acust. Acta Acust. 91, 312-325 (2005).

${ }^{11}$ V. Välimäki, J. Pakarinen, C. Erkut, and M. Karjalainen, "Discrete-time modelling of musical instruments," Rep. Prog. Phys. 69, 1-78 (2006).

${ }^{12}$ J.-M. Adrien, "The missing link: Modal synthesis," in Representations of Musical Signals, edited by G. De Poli, A. Piccialli, and C. Roads (MIT Press, Cambridge, MA, 1991), pp. 269-297.

${ }^{13}$ R. Rabenstein and L. Trautmann, "Digital sound synthesis of string instruments with the functional transformation method," Signal Process. 83, 1673-1688 (2003).

${ }^{14}$ L. Trautmann and R. Rabenstein, Digital Sound Synthesis by Physical Modeling Using the Functional Transformation Method (Kluwer Academic/Plenum Publishers, New York, 2003), Chap. 5, pp. 95-130.

${ }^{15} \mathrm{~S}$. Bilbao, "Modal type synthesis techniques for nonlinear strings with an energy conservation property," in Proceedings of the International Conference on Digital Audio Effects (DAFx-04), Naples, Italy (2004), pp. 119-124.

${ }^{16} \mathrm{~S}$. Bilbao, "Energy-conserving finite difference schemes for tensionmodulated strings," in Proceedings on the IEEE International Conference on Acoustics, Speech and Signal Processing, Montreal, Quebec (2004), pp. 285-288.

${ }^{17}$ S. A. Van Duyne and J. O. Smith III, "The 2-D digital waveguide mesh," in Proceedings of the IEEE Workshop on Applied Signal Processing to Audio and Acoustics (WASPAA93), New Paltz, NY (1993), pp. 177-180.

${ }^{18} \mathrm{~F}$. Fontana and D. Rocchesso, "Signal-theoretic characterization of waveguide mesh geometries for models of two-dimensional wave propagation in elastic media," IEEE Trans. Speech Audio Process. 9, 152-161 (2001).

${ }^{19}$ L. Savioja and V. Välimäki, "Interpolated rectangular 3-D digital waveguide mesh algorithms with frequency warping," IEEE Trans. Speech Audio Process. 11, 783-790 (2003).
${ }^{20} \mathrm{~S}$. Petrausch and R. Rabenstein, "Tension modulated nonlinear $2 \mathrm{D}$ models for digital sound synthesis with the functional transformation method," in Proceedings of the European Signal Processing Conference (EUSIPCO2005), Antalya, (2005).

${ }^{21}$ C. H. Jenkins and J. W. Leonard, "Nonlinear dynamic response of membranes: State of the art," Appl. Mech. Rev. 44, 319-328 (1991).

${ }^{22}$ C. H. Jenkins, "Nonlinear dynamic response of membranes: State of the art—update," Appl. Mech. Rev. 48, S41-S48 (1996).

${ }^{23}$ J. S. Rao, Dynamics of Plates (Marcel Dekker, New York, 1999), Chap. 6, pp. 179-227.

${ }^{24}$ S. Bilbao, "Sound synthesis for nonlinear plates," in Proceedings of the International Conference on Digital Audio Effects (DAFx-05) Madrid (2005), pp. 243-248.

${ }^{25}$ S. Bilbao, "A family of conservative finite difference schemes for the dynamical von Karman plate equations," Numer. Methods Partial Differ. Equ. 24, 193-216 (2008).

${ }^{26} \mathrm{~F}$. Avanzini and R. Marogna, "A modular physically-based approach to the sound synthesis of membrane percussion instruments," IEEE Trans. Audio Speech Lang. Process. 18, 891-902 (2010).

${ }^{27}$ R. Marogna and F. Avanzini, "Physically based synthesis of nonlinear circular membranes," in Proceedings of the International Conference on Digital Audio Effects (DAFx-09) Como (2009), pp. 373-379.

${ }^{28}$ H. M. Berger, "A new approach to the analysis of large deflections of plates," ASME J. Appl. Mech. 22, 465-472 (1955).

${ }^{29}$ Zs. Garamvölgyi, "Physics-based modeling of membranes for sound synthesis applications," Master's thesis, Budapest University of Technology and Economics, Hungary (2008).

${ }^{30} \mathrm{~B}$. Bank, "Energy-based synthesis of tension modulation in strings," in Proceedings of the International Conference on Digital Audio Effects (DAFx-09) Como (2009), pp. 365-372.

${ }^{31}$ R. Marogna, F. Avanzini, and B. Bank, "Energy based synthesis of tension modulation in membranes," in Proceedings of the International Conference on Digital Audio Effects (DAFx-10) Graz (2010), pp. 102-108.

${ }^{32}$ A. Chaigne and A. Askenfelt, "Numerical simulations of piano strings. I. A physical model for a struck string using finite difference methods," J. Acoust. Soc. Am. 95, 1112-1118 (1994).

${ }^{33}$ S. Dahl, "Playing the accent—comparing striking velocity and timing in an ostinato rhythm performed by four drummers," Acust. Acta Acust. 90, 762-776 (2004)

${ }^{34}$ N. H. Fletcher and T. D. Rossing, The Physics of Musical Instruments (Springer-Verlag, New York, 1991), Chap. 2, p. 38.

${ }^{35}$ N. H. Fletcher and T. D. Rossing, The Physics of Musical Instruments (Springer-Verlag, New York, 1991), Chap. 3, pp. 66-70.

${ }^{36}$ K. A. Legge and N. H. Fletcher, "Nonlinear generation of missing modes on a vibrating string,” J. Acoust. Soc. Am. 76, 5-12 (1984).

${ }^{37}$ P. M. Morse, Vibration and Sound, 2nd edition (McGraw-Hill, New York, 1948), Chap. 3, pp. 89-91.

${ }^{38} \mathrm{X}$. Boutillon, "Model for piano hammers: Experimental determination and digital simulation,” J. Acoust. Soc. Am. 83, 746-754 (1988).

${ }^{39}$ L. Rhaouti, A. Chaigne, and P. Joly, "Time-domain modeling and numerical simulation of a kettledrum," J. Acoust. Soc. Am. 105, 3545-3562 (1999).

${ }^{40}$ K. H. Hunt and F. R. E. Crossley, "Coefficient of restitution interpreted as damping in vibroimpact," ASME J. Appl. Mech. 42, 440-445 (1975)

${ }^{41}$ A. Stulov, "Hysteretic model of the grand piano felt," J. Acoust. Soc. Am. 97, 2577-2585 (1995). 